Erratum

\title{
Cytosine deaminase as a negative selective marker for Arabidopsis
}

\author{
Ranjan J. Perera, Christian G. Linard ${ }^{1}$ and Ethan R. Signer*
}

Plant Molecular Biology 23: 793-799, 1993.

There are two errors in Fig. 3: first, the lower left-panel was printed upside down and second, the placement of the panels with respect to their corresponding references in the legend was incorrect. The whole figure should have appeared as follows:
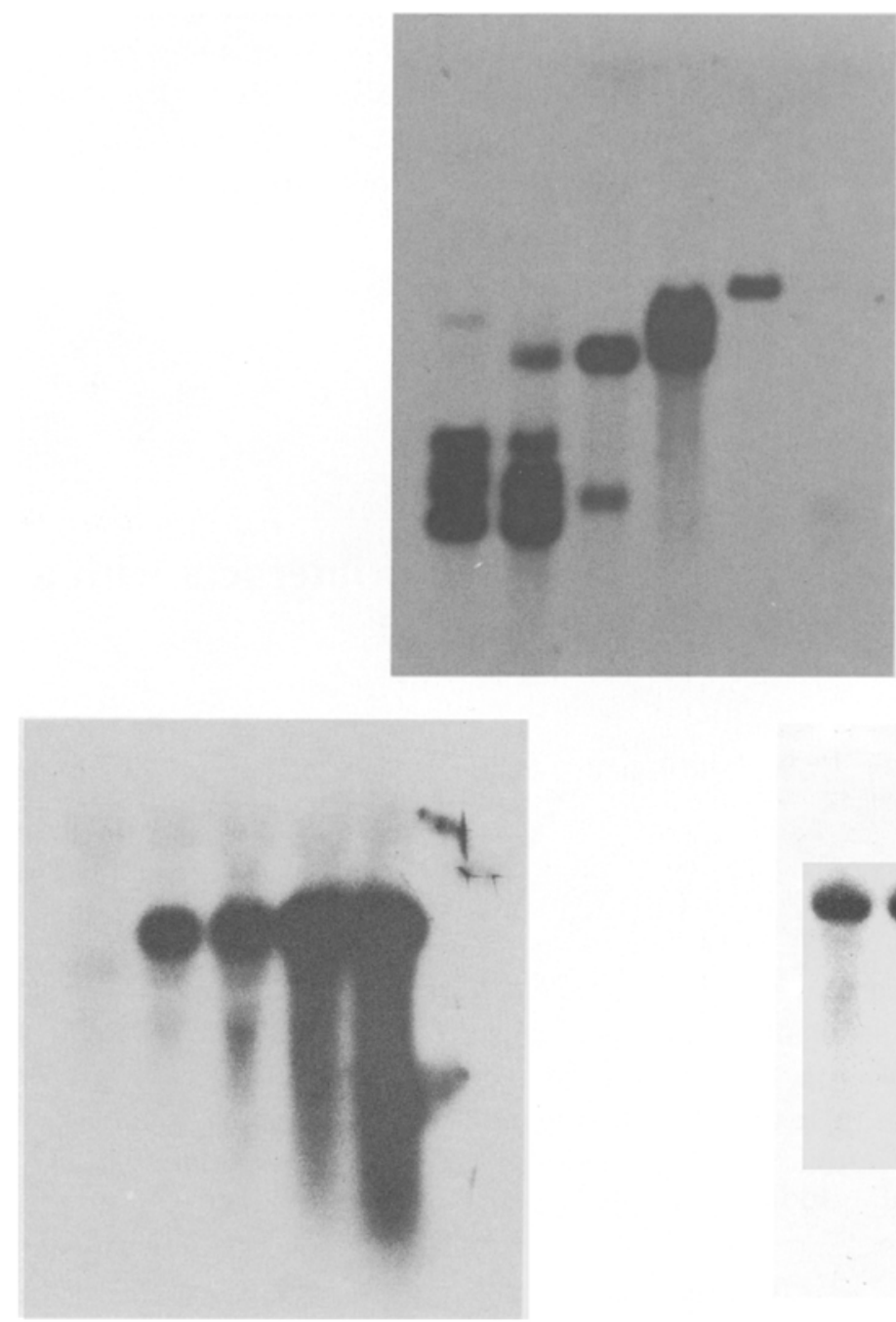

Fig. 3. Molecular analysis of representative transgenic lines. Top: Southern analysis; lanes (from left): CDD1-2A, CDD2-2C, CDD3-2C, CDD4-2A, CDD5-2B, CDR1-21; probe: codA. Bottom: northern analysis; lanes (from left): CDR1-21, CDD1-2A, CDD2-2C, CDD3-2C, CDD4-2A; probes; bottom left, $\operatorname{cod} A$; bottom right, $A B T$ ( $\beta$-tubulin). 